\title{
Bounds for solutions of a class of quasilinear elliptic boundary value problems in terms of the torsion function
}

\author{
L. E. Payne $\dagger$ \\ Forschungsinstitut für Mathematik ETH, Zürich and Cornell University, \\ Ithaca, N.Y., U.S.A.
}

(MS received 17 March 1980, Revised MS received 10 July 1980)

\section{Synopsis}

In this paper maximum principles are employed to relate solutions of certain classes of nonlinear elliptic problems to solutions of the associated torsion problem. By this method a number of new isoperimetric inequalities are derived. In special cases solutions of the nonlinear problems are also related to solutions of the clamped membrane problem.

\section{Introduction}

In this paper we make use of the maximum principle to compare solutions of a class of nonlinear elliptic boundary value problems with the solutions of simpler linear problems defined on the same domain. In some cases the methods lead to isoperimetric results.

Let $D$ be a bounded convex domain in $R_{N}$ with $C^{2+\alpha}$ boundary $\partial D$. We shall be concerned in this paper with positive solutions of the following boundary value problem defined on $D$ :

$$
\begin{array}{rll}
\Delta u+\lambda f(u)=0 & \text { in } & D \\
u=0 & \text { on } & \alpha D .
\end{array}
$$

Here $\Delta$ is the Laplace operator and $\lambda$ is a constant. It is known of course that positive solutions do not necessarily exist for all values of $\lambda$. Also for strictly nonlinear $f$, more than one positive solution may correspond to the same value of $\lambda$. Sufficient conditions for existence and for uniqueness appear in the literature (see e.g. Amann [1] and the references cited therein).

For simplicity we shall assume throughout that

$$
f(u) \geqq 0, \quad f^{\prime}(u) \geqq 0
$$

for positive solutions of (1.1). In a number of cases these constraints can be relaxed, but in order to make the already computationally involved calculations easier to follow we shall impose (1.2) in our subsequent discussions.

The principal purpose of this paper is to compare solutions of (1.1) with 
solutions of the simple linear problem

$$
\begin{array}{rll}
\Delta \psi+1=0 & \text { in } & D \\
\psi=0 & \text { on } & \partial D .
\end{array}
$$

The special case $f(u) \equiv u, N=2$, will lead to interesting new isoperimetric inequalities of the type indicated by Hersch [4] (see (3.12) and (5.15)). Because of the physical interpretation of (1.3) for $N=2$, we refer to $\psi$ as the torsion function.

In our arguments we make extensive use of the maximum principles of the type derived by Payne [6], and by Stakgold and Payne [9], [11]. Since these principles are dependent on the results of [5], we require that $f$ be a $C^{1}$ function of its argument and that $u$ be a classical solution of (1.1). In fact we shall assume throughout that $u \in C^{2}(\bar{D})$ whenever it exists.

It should be noted that all of the results of this paper actually hold for somewhat more general domains. It is only necessary that the mean curvature at every point of $D$ be nonnegative.

\section{Statement of maximum principles}

The following maximum principles derived in [6], [11] will be employed throughout this paper:

Principle I. Let $u$ be a positive classical solution of (1.1) in a convex region $D$, where $f \in C^{1}[0, \infty)$ satisfies (1.2); then at points in $D$

$$
|\operatorname{grad} u|^{2} \leqq 2 \lambda\left[F\left(u_{M}\right)-F(u)\right]
$$

where

$$
F(u)=\int_{0}^{u} f(\eta) d \eta, \quad u_{M}=\max _{\bar{D}} u(x) .
$$

Principle II. Let $\psi$ be the solution of (1.3) in a convex region $D$, then at points in $D$

$$
|\operatorname{grad} \psi|^{2}+\frac{2}{N} \psi \leqq \max _{\partial D}|\operatorname{grad} \psi|^{2} .
$$

Generalizations of PI and PII appear in [7] and [8].

It should be pointed out that (2.1) is an optimal inequality, the equality sign holding in the limit for a spheroidal domain with axis of finite length as the radius of the meridian section tends to infinity. We shall subsequently refer to this limiting domain as the infinite slab.

\section{Bounds for $\psi$ in terms of the solution of (1.1)}

We first establish the following theorem:

THEOREM I. Let $u$ be a classical positive solution of (1.1), (1.2) in a convex region $D$ and set

$$
\tilde{\phi}=\left[\int_{0}^{u_{M}} \frac{d \eta}{\sqrt{F\left(u_{M}\right)-F(\eta)}}\right]^{2}-\left[\int_{u}^{u_{M}} \frac{d \eta}{\sqrt{F\left(u_{M}\right)-F(\eta)}}\right]^{2},
$$


then the following inequalities hold:

and

$$
\tilde{\phi} \leqq 4 \lambda \psi \quad \text { in } D
$$

$$
\frac{1}{\sqrt{F\left(u_{M}\right)}} \int_{0}^{u_{M}} \frac{d \eta}{\sqrt{F\left(u_{M}\right)-F(\eta)}}|\operatorname{grad} u| \leqq 2 \lambda|\operatorname{grad} \psi| \text { on } \partial D .
$$

The motivation for the specific choice for $\tilde{\phi}$ is as follows: We are seeking inequalities which are sharp in the sense that the equality signs should hold in the limit as $D$ degenerates to the infinite slab, i.e. for the solution of (1.1) for $N=1$. But the right hand side of (3.1) is just a multiple of the solution of (1.3) in one space dimension.

To establish (3.2) and (3.3) we show that $\varphi$ satisfies

$$
\Delta \tilde{\varphi}+4 \lambda \geqq 0 \text { in } D \text {. }
$$

Since $\tilde{\varphi}$ vanishes on $\partial D,(3.2)$ follows immediately from the classical maximum principle for subharmonic functions.

A direct calculation gives for $u \neq u_{M}$

$$
\begin{aligned}
\Delta \tilde{\varphi}+4 \lambda=\left[2\left[F\left(u_{M}\right)-F(u)\right]^{\frac{1}{2}}-f(u) \int_{u}^{u_{M}} \frac{d \eta}{\sqrt{F\left(u_{M}\right)-F(\eta)}}\right] \\
\times\left[\frac{2 \lambda\left[F\left(u_{M}\right)-F(u)\right]-|\operatorname{grad} u|^{2}}{\left[F\left(u_{M}\right)-F(u)\right]^{\frac{3}{2}}}\right] .
\end{aligned}
$$

The second term on the right is clearly nonnegative by (2.1). To check the sign of the first term we set

$$
R(u)=2\left[F\left(u_{M}\right)-F(u)\right]^{\frac{1}{2}}-f(u) \int_{u}^{u_{M}} \frac{d \eta}{\sqrt{F\left(u_{M}\right)-F(\eta)}}
$$

and form

$$
\frac{d R}{d u}=-f^{\prime}(u) \int_{u}^{u_{M}} \frac{d \eta}{\sqrt{F\left(u_{M}\right)-F(\eta)}} \leqq 0,
$$

the last inequality following from (1.2). An integration yields

$$
R(u) \geqq R\left(u_{M}\right)=0,
$$

which establishes (3.4) and thus (3.2) once we observe that as $u \rightarrow u_{M}$, both grad $\tilde{\varphi}$ and the right hand side of (3.5) tend to zero.

Inequality (3.3) follows from the well known fact that since $\tilde{\varphi} \leqq 4 \lambda \psi$ in $D$ and $\tilde{\varphi}=\psi=0$ on $\partial D$ then at each point of the boundary $|\operatorname{grad} \tilde{\varphi}| \leqq 4 \lambda|\operatorname{grad} \psi|$ (see e.g. [10]). Bounds for $\max _{\partial \mathrm{D}}|\operatorname{grad} \psi|$ may be found in [3] and [6].

EXAMPLES. (A) Clamped membrane problem: $f(u) \equiv u$.

The first eigenfunction (with corresponding eigenvalue $\lambda_{1}$ ) in the clamped membrane problem for $D$ satisfies

$$
\begin{array}{rll}
\Delta w+\lambda_{1} w=0 & \text { in } \quad D \\
w=0 & \text { on } & \partial D, \quad w>0 \quad \text { in } D .
\end{array}
$$


In this case (3.2) becomes

$$
\frac{\pi^{2}}{4}-\left[\cos ^{-1} \frac{w}{w_{M}}\right]^{2} \leqq 2 \lambda_{1} \psi \quad \text { in } \quad D
$$

while (3.3) leads to

$$
\frac{\pi}{2}|\operatorname{grad} w| \leqq \lambda_{1} w_{M}|\operatorname{grad} \psi| \text { on } \partial D .
$$

In particular (3.10) yields

$$
\lambda_{1} \psi_{M} \geqq \pi^{2} / 8, \quad \psi_{M} \equiv \max _{D} \psi
$$

with the equality sign holding in the limit for an infinite slab. Other isoperimetric inequalities involving the volume of $D$ and the Dirichlet integral of $u$ over $D$ follow from the above results.

(B) The case $f(u)=e^{u}$.

From (3.2) we obtain for this example

$$
e^{-u_{M} / 2} \ln \left[e^{u_{M} / 2}+\sqrt{e^{u_{M}}-1} \leqq \sqrt{\lambda \psi_{M}}\right.
$$

or

$$
e^{-u_{M} / 2} \cosh ^{-1}\left[e^{u_{M} / 2}\right] \leqq \sqrt{\lambda \psi_{M}} .
$$

It is well known that in this case there is a critical value of $\lambda$ (call it $\lambda_{c}$ ) such that for $\lambda>\lambda_{c}$ no solution exists, while if $\lambda<\lambda_{c}$ more than one solution will exist (see e.g. Bandle [2]). Inequality (3.13) is again sharp in that the equality sign holds in the limit as $D$ degenerates to an infinite slab. Of course for certain geometries sharper inequalities can be obtained using monotonicity and symmetrization arguments. Results in this direction will appear in a forthcoming paper of Bandle.

\section{Other inequalities relating $\psi$ to the solution of (1.1) for $N=1$}

In this section we assume $\lambda^{*}$ and $f$ to be such that at least one solution exists to the one dimensional problem

$$
\begin{aligned}
v^{\prime \prime}+\lambda^{*} f(v) & =0, \quad 0 \leqq x \leqq a \\
v^{\prime}(0) & =v(a)=0,
\end{aligned}
$$

where $\lambda^{*}$ and $a$ are chosen to satisfy one of the following sets of conditions, i.e.

$$
a \geqq \sqrt{2 \psi_{\mathrm{M}}}
$$

or

$$
\begin{gathered}
\lambda=\lambda^{*} \\
v(0) \geqq \max _{D} u(x) .
\end{gathered}
$$

It turns out that both of the conditions in (4.3) will be required for examples like 
$f(u)=u^{q}, q>1$, where, for fixed $\lambda$, a solution of (4.1) exists for any choice of $a$. Clearly the second condition in (4.3) may be difficult to verify; however, one can often obtain crude bounds for the maximum value of the unknown solution.

Before proceeding, let us recall that one simple criterion on $f$ which will insure that there is at most one positive solution of (1.1), (1.2) is that for $\xi, \eta \in[0, \infty)$, $\xi \neq \eta$ the following inequality holds

$$
(\xi-\eta)\left(\frac{f(\xi)}{\xi}-\frac{f(\eta)}{\eta}\right)<0
$$

(see [11]).

Now let $v$ be a positive solution of $(4.1),(4.2)$ or of (4.1), (4.3). We define

$$
\varphi(x)=v\left(\sqrt{2\left(\psi_{M}-\psi\right)}\right) .
$$

If (4.2) holds then $\varphi$ is defined in all of $D$. Otherwise it is defined on the subdomain of $D$ given by $\sqrt{2\left(\psi_{M}-\psi\right)} \leqq a$. If $v$ satisfies (4.1), (4.2) then $\varphi \geqq 0$ on $\partial D$ and hence in $D$.

We now establish the following lemma:

LEMMA I. Let $\varphi$ be given by (4.5) where $v$ is a solution of (4.1); then

$$
\Delta \varphi+\lambda^{*} f(\varphi) \leqq 0 \text { in } D^{*}-\left\{P_{1}\right\}
$$

where $P_{1}$ is the unique point in $D$ at which $\psi$ assumes its maximum value and $D^{*} \equiv D$ if $a \geqq \sqrt{2 \psi_{M}}$; otherwise $D^{*}$ is the subregion defined by $\sqrt{2\left(\psi_{M}-\psi\right)} \leqq a$.

The proof of the lemma again makes use of PI and the particular form of $\varphi$. We compute as follows for $\psi \neq \psi_{M}$ :

$$
\begin{aligned}
\Delta \varphi+\lambda^{*} f(\varphi) & \left.=\frac{1}{\sqrt{2\left(\psi_{M}-\psi\right)}}\left[\lambda^{*} \sqrt{2\left(\psi_{M}-\psi\right.}\right) f(v)+v^{\prime}\right]\left[1-\frac{1}{2} \frac{|\operatorname{grad} \psi|^{2}}{\psi_{M}-\psi}\right] \\
& =\frac{1}{\sqrt{2\left(\psi_{M}-\psi\right)}} R\left(\sqrt{2\left(\psi_{M}-\psi\right.}\right) S(\psi) .
\end{aligned}
$$

The right hand side in fact tends to zero as $\psi \rightarrow \psi_{\mathrm{M}}$.

In (4.7) $S$ is clearly nonnegative by PI. To show that $R$ is nonpositive we form

$$
\frac{d R}{d x}(x)=\lambda^{*} x f^{\prime}(x) v^{\prime}
$$

But $v^{\prime}(x)$ is clearly nonpositive in $D^{*}$ since $v^{\prime \prime}(x) \leqq 0$ and $v^{\prime}(0)=0$. We thus conclude that in $D^{*}$

$$
R(x) \leqq R(0)=0 .
$$

This proves the lemma.

We see then that if $v$ satisfies (4.1), (4.3) and $\varphi \geqq 0$ in $D$, then $\varphi$ is an upper solution of $(1.1)$, (1.2), i.e.

$$
0 \leqq u \leqq \varphi .
$$

Note that (4.5) and (4.3) imply that $u\left(P_{1}\right) \leqq \phi\left(P_{1}\right)$. We shall show that with 
appropriate assumptions on $f,(4.10)$ is impossible and thus that (4.2) cannot hold. We now establish the following theorem:

THEOREM II. Let (1.1), (1.2) possess a unique positive classical solution. Suppose further that (4.1), (4.2) possesses a positive solution for $f$ satisfying

$$
(\xi-\eta)\left[\frac{f(\xi)}{\xi}-\frac{f(\eta)}{\eta}\right] \leqq 0, \quad \xi, \eta \in[0, \infty),
$$

then either

$$
u \leqq \varphi \quad \text { in } \quad D
$$

or

$$
\lambda>\lambda^{*} \text {. }
$$

If on the other hand (4.1), (4.3) has a positive solution and $f$ satisfies

$$
(\xi-\eta)\left[\frac{f(\xi)}{\xi}-\frac{f(\eta)}{\eta}\right] \geqq 0, \quad \xi, \eta \in[0, \infty),
$$

then

$$
a \leqq \sqrt{2 \psi_{M}} .
$$

The proof in both cases is established by contradiction. To prove the first part of the theorem we assume that in some subdomain $D_{1} \subset D, u>\varphi$. We then form

$$
\begin{aligned}
& \int_{D_{1}}\left[(u-\varphi) \Delta(u-\varphi)-\frac{(u-\varphi)^{2}}{u} \Delta u\right] d x \\
& \quad \geqq \lambda^{*} \int_{D_{1}} \varphi(u-\varphi)\left(\frac{f(\varphi)}{\varphi}-\frac{f(u)}{u}\right) d x+\left(\lambda^{*}-\lambda\right) \int_{D_{1}} \varphi(u-\varphi) \frac{f(u)}{u} d x,
\end{aligned}
$$

where we have used (4.6). On applying the divergence theorem on the left, simplifying, and regrouping terms we obtain

$$
\begin{aligned}
-\int_{D_{1}} u^{2}\left|\operatorname{grad}\left(\frac{\varphi}{u}\right)\right|^{2} d x-\lambda^{*} \int_{D_{1}} \varphi(u-\varphi) & \left(\frac{f(\varphi)}{\varphi}-\frac{f(u)}{u}\right) d x \\
& \geqq\left(\lambda^{*}-\lambda\right) \int_{D_{1}} \varphi(u-\varphi) \frac{f(u)}{u} d x .
\end{aligned}
$$

Note that $D_{1}$ is a strict subdomain of $D$ if the inequality sign holds in (4.2). Also the integrals may be interpreted in the limiting sense if $P_{1} \in D_{1}$, since $|\operatorname{grad} \varphi|$ is bounded in $D$. Unless $\varphi / u \equiv$ constant the left hand side is negative. But the ratio $\varphi / u$ cannot be constant if $D$ is a bounded region; thus the first part of the theorem is established.

To prove the second part of the theorem, we need merely show that $\varphi$ cannot be positive throughout $D$. Suppose $\varphi>0$ in $D$ and thus that (4.10) holds. Then

$$
\int_{D}\left[u \Delta u-\frac{u^{2}}{\varphi} \Delta \varphi\right] d x \geqq \lambda \int_{D} u^{2}\left[\frac{f(\varphi)}{\varphi}-\frac{f(u)}{u}\right] d x .
$$


An integration by parts on the left gives

$$
-\int_{D}\left|\operatorname{grad} u-\frac{u^{2}}{\varphi} \operatorname{grad} \varphi\right|^{2} d x-\lambda \int_{D} u^{2}\left[\frac{f(\varphi)}{\varphi}-\frac{f(u)}{u}\right] d x \geqq 0 .
$$

In view of (4.14) the inequality (4.19) can hold if and only if $f(\varphi) / \varphi=$ constant throughout $D$, but in this case it is well known that $\varphi$ cannot remain positive in $\bar{D}$. We are thus led to a contradiction which shows that $\varphi$ must vanish in $\bar{D}$. Because of the form of $\varphi$, this implies (4.15), and the theorem is established.

If we apply Theorem II to the clamped membrane problem with $v$ chosen to satisfy (4.3), we are led again to (3.12). Note that in this case (4.3) is easily satisfied since any constant multiple of a solution is still a solution. On the other hand if we choose $f(u)=e^{u}$ and use the first half of Theorem II which holds for $\max _{D} u \leqq 1$, we conclude that in this case either $u \leqslant \varphi$ in $D$ or else $\lambda>\lambda^{*}$.

If the second part of Theorem II is applicable, and if the one dimensional problem (4.1), (4.3) can be solved explicitly, then one can represent $a$ as

$$
a=g(\lambda) \text {. }
$$

the conclusion (4.15) states that

$$
g(\lambda) \leqq \sqrt{2 \psi_{M}}
$$

On the other hand if (4.1) can be solved explicitly with the equality sign in (4.2) and $\lambda=\lambda^{*}$, then according to the theorem

$$
0 \leqq u \leqq \varphi \text { in } D \text {. }
$$

For instance it is easily checked that the solution of

$$
\begin{aligned}
v^{\prime \prime}+\lambda^{*} e^{v} & =0 \quad 0 \leqq x \leqq a \\
v^{\prime}(0), v(a) & =0
\end{aligned}
$$

is given by

$$
v=2 \log \left[\sqrt{2 k} \operatorname{sech}\left(k \sqrt{\lambda^{*} x}\right)\right]
$$

where $k$ must be chosen to satisfy

$$
\sqrt{2 k}=\cosh \left(k \sqrt{\lambda^{*} a}\right) .
$$

Clearly (4.25) can hold only for sufficiently small values of $\lambda^{*} a^{2}$. If, however, the problem has a solution for $\lambda^{*}=\lambda$ and $a=\sqrt{2 \psi_{M}}$ we may apply Theorem II as follows: Let $v$ be a solution of

$$
\begin{array}{rlrl}
v^{\prime \prime}+\lambda e^{v} & =0 & 0 \leqq x \leqq \sqrt{2 \psi_{M}} \\
v^{\prime}(0) & =0, & v\left(\sqrt{2 \psi_{M}}\right)=0 .
\end{array}
$$

Then any solution of (1.1) with $f(u)=e^{u}$, which satisfies

$$
\max _{D} u \leqq 1
$$

must also satisfy

$$
0 \leqq u \leqq \varphi \text { in } \quad D .
$$


It thus follows that if $u$ satisfies (4.27), then on $\partial D$

$$
\begin{aligned}
|\operatorname{grad} u|^{2} & \leqq\left|v^{\prime}\right|^{2} \frac{|\operatorname{grad} \psi|^{2}}{2 \psi_{M}} \\
& \leqq 2 \lambda\left(2 k^{2}-1\right)
\end{aligned}
$$

For instance if

$$
\lambda \psi_{M}=\operatorname{sech}^{2} 1
$$

then

$$
k^{2}=\left[2 \lambda \psi_{\mathrm{M}}\right]^{-1},
$$

and we find that in $D$

$$
0 \leqq u \leqq 2 \log \left[\cosh 1 / \cosh \sqrt{1-\psi / \psi_{M}}\right],
$$

and on $\partial D$

$$
|\operatorname{grad} u|^{2} \leqq 2 \lambda \sinh ^{2} 1 \equiv 2\left[\psi_{M}\right]^{-1} \tanh ^{2} 1 .
$$

The inequalities above are best possible for the particular choice of $\lambda \psi_{M}$ given by (4.31), the equality signs holding in the limit as the domain degenerates to the infinite slab.

To give another illustration of how the second half of Theorem II can be applied, we consider the problem

$$
\begin{array}{rll}
\Delta u+\lambda u^{3}=0 & \text { in } & D \subset R^{2} \\
u=0 & \text { on } & \partial D .
\end{array}
$$

Such an equation has a positive solution corresponding to any positive value of $\lambda$. We now define the Stekloff constant $\Omega$ by

$$
\Omega=\min _{\substack{h=0 \text { on } \partial D \\ h \in c^{1}(D)}} \frac{\left[\int_{D}|\operatorname{grad} h|^{2} d x\right]^{2}}{\int_{D} h^{4} d x} .
$$

The minimizing function $w$ for (4.36) satisfies

$$
\begin{gathered}
\Delta w+\frac{\Omega w^{3}}{\int_{D}|\operatorname{grad} w|^{2} d x}=0 \text { in } D \\
w=0 \text { on } \partial D .
\end{gathered}
$$

Let us normalize $w$ by the condition

$$
\int_{D}|\operatorname{grad} w|^{2} d x=\Omega / \lambda .
$$

Then $w \equiv u$, and

$$
\int_{D} u^{4} d x=\Omega / \lambda^{2}
$$


We now represent $u$ at a point $P$ in $D$ in terms of the Green's function $G(P, Q)$ by the expression

$$
u(P)=\lambda \int_{D} G(P, Q) u^{3}(Q) d x,
$$

and make use of Holder's inequality to obtain

$$
u(P) \leqq \lambda\left\{\int_{D}[G(P, Q)]^{4} d x\right\}^{\frac{1}{4}}\left\{\int_{D} u^{4} d x\right\}^{\frac{3}{4}}
$$

An application of a result of Weinberger [12] then yields

$$
u_{M} \leqq \frac{1}{4 \pi} \frac{\Omega^{\frac{3}{4}}}{\lambda^{\frac{1}{2}}}(24 A)^{\frac{1}{4}}
$$

where $A$ is the area of $D$.

The corresponding one dimensional problem is

$$
\begin{aligned}
v^{\prime \prime}+\lambda^{*} v^{3}=0, & 0 \leqq x \leqq a \\
v^{\prime}(0)=0, & v(a)=0
\end{aligned}
$$

from which we easily compute

$$
v_{M}=\sqrt{\frac{2}{\lambda^{*}}} \frac{\gamma}{a}
$$

where

$$
\gamma=\int_{0}^{1} \frac{d t}{\sqrt{1-t^{4}}}
$$

Conditions (4.3) are thus satisfied if we set $\lambda^{*}=\lambda$ and choose $a$ to satisfy

$$
a=\frac{4 \pi \gamma}{\Omega^{\frac{3}{4}}(6 A)^{\frac{1}{4}}}
$$

It follows then from the second part of Theorem II that

$$
\Omega^{3} A \psi_{M}^{2} \geqq 32 \pi^{4} \gamma^{4} / 3
$$

a rather crude inequality, but one which can be sharpened if a better estimate for $u_{M}$ can be computed. Note that we have obtained lower bounds for $\Omega$ and hence by (4.38) and (4.39) lower bounds for $\int_{D}|\operatorname{grad} u|^{2} d x$ and $\int_{D} u^{4} d x$. Upper bounds for these expressions are easily computed using (4.36).

\section{Inequalities relating $\boldsymbol{\psi}$ to the solution of (1.1) for the $\mathbf{N}$-ball}

We now suppose that $\lambda^{*}$ and $f$ are such that a classical positive solution of

$$
\begin{array}{rlrl}
\frac{d^{2} \chi}{d r^{2}}+\frac{(N-1)}{r} \frac{d \chi}{d r}+\lambda^{*} f(\chi) & =0, & 0 \leqq r \leqq R \\
\chi^{\prime}(0) & =0, \quad \chi(R)=0
\end{array}
$$


exists, where $\lambda^{*}$ and $R$ are chosen to satisfy one of the following sets of conditions:

$$
R \geqq N \sigma_{M}
$$

or

$$
\begin{gathered}
\lambda=\lambda^{*} \\
\chi(0) \geqq \max _{D} u .
\end{gathered}
$$

In (5.2) we have used the notation

$$
\sigma_{M}=\max _{\partial D}|\operatorname{grad} \psi| \equiv \max _{\bar{D}}|\operatorname{grad} \psi| .
$$

We define a function $\Phi$ as follows:

$$
\left.\Phi=\chi\left(\sqrt{N\left(N \sigma_{M}^{2}-2 \psi\right.}\right)\right) \text { in } D^{*}
$$

where $\chi$ is a positive solution of (5.1), (5.2) or of (5.1), (5.3) and $D^{*} \equiv D$ if (5.2) holds. If $R<N \sigma_{M}$ then $D^{*}$ is the subregion of $D$ defined by $N\left(N \sigma_{M}^{2}-2 \psi\right) \leqq R^{2}$. Again if $\chi$ satisfies (5.1), (5.2) then $\Phi \geqq 0$ on $\partial D$ and therefore in $D$.

Let us now establish the analogue of Lemma I, again choosing the origin $P_{1}$ at the point at which $\psi=\psi_{M}$.

LEMMA II. Let $\Phi$ be given by (5.5) where $\chi$ is a solution of (5.1) then

$$
\Delta \Phi+\lambda^{*} \Phi \leqq 0 \text { in } D^{*}-\left\{P_{1}\right\}
$$

A direct computation gives in $D_{1}-\left\{P_{1}\right\}$

$$
\begin{aligned}
\Delta \Phi+\lambda^{*} \Phi & =\frac{1}{\tau^{N}}\left[\lambda^{*} \tau^{N} f(\chi)+N \tau^{N-1} \chi^{\prime}\right]\left[1-\frac{|\operatorname{grad} \psi|^{2}}{\sigma_{M}^{2}-2 N^{-1} \psi}\right] \\
& =\frac{1}{\tau^{N}} R_{1}(\tau) S_{1}(\psi)
\end{aligned}
$$

where we have used the notation

$$
\tau=\sqrt{N\left(N \sigma_{M}^{2}-2 \psi\right)} .
$$

We observe again that the right hand side of (5.7) tends to zero in the limit as $\tau \rightarrow 0$. However, it follows easily from the proof of (2.4) that the quantity $N \sigma_{M}^{2}-2 \psi_{M}$ is strictly positive unless $D$ is the $N$-ball. Thus the parameter $\tau$ is restricted to the interval

$$
\sqrt{N\left(N \sigma_{M}^{2}-2 \psi_{M}\right)} \leqq \tau \leqq \sqrt{N\left(N \sigma_{M}^{2}-2 \psi_{2}\right)}
$$

where $\psi_{2}$ is given by $N\left(N \sigma_{M}^{2}-2 \psi_{2}\right)=R^{2}$ if $D^{*}$ is a proper subdomain of $D$; otherwise it is zero.

In (5.7) we note that $S_{1}$ is nonnegative (by (2.4)). To determine the sign of $R$, we observe that

$$
\frac{d R}{d \tau}=\lambda^{*} \tau^{N} f^{\prime} \frac{d \chi}{d \tau} .
$$


Again since $\frac{d}{d \tau}\left(\tau^{N-1} \frac{d \chi}{d \tau}\right) \leqq 0$ we conclude that $\frac{d \chi}{d \tau}$ is nonpositive and hence that $R \leqq 0$ in $D^{*}$. Thus the lemma is proved.

We remark again that if $\chi$ satisfies (5.1), (5.3) and $\Phi \geqq 0$ on $\partial D$ then $\Phi$ is an upper solution, i.e.

$$
0 \leqq u \leqq \Phi .
$$

With the aid of Lemma II we now establish the following:

THEOREM III. Let (1.1), (1.2) possess a unique positive classical solution. Suppose further that (5.1), (5.2) has a positive solution for $f$ satisfying (4.11) then either

$$
u \leqq \Phi \text { in } D
$$

or

$$
\lambda \geqq \lambda^{*} \text {. }
$$

On the other hand if (5.1), (5.3) has a positive solution and $f$ satisfies (4.14), then

$$
R \leqq N \sigma_{M}
$$

Again the proof is made by contradiction; in fact the method is precisely the same as that used in the proof of Theorem II. We do not repeat it here.

Again if we apply Theorem III in the case of the clamped membrane $(N=2)$ we are led to the isoperimetric inequality

$$
2 \sqrt{\lambda_{1}} \sigma_{M} \geqq j_{0},
$$

where $j_{0}$ is the first root of the Bessel function $J_{0}(x)$.

As we noted in the previous section, if the solution of (5.1), (5.3) could be determined explicitly, one could represent $R$ as

$$
R=h(\lambda) \text {. }
$$

Then (5.14) would become

$$
h(\lambda) \geqq N \sigma_{\mathrm{M}} .
$$

We remark that a somewhat sharper but more complicated inequality could actually be derived if we used instead of (5.1) the problem

$$
\begin{array}{rlrl}
\frac{d^{2} \chi}{d r^{2}}+\frac{(N-1)}{r} \frac{d \chi}{d r}+\lambda^{*} f(\chi) & =0, & \alpha \leqq r \leqq R \\
\chi^{\prime}(\alpha) & =0, \quad \chi(R)=0
\end{array}
$$

where $\alpha=\sqrt{N\left(N \sigma_{M}^{2}-2 \psi_{M}\right)}$.

The results of this section remain valid for nonconvex $D$.

\section{Some additional results}

In Section III we derived results which gave an upper bound for $\int_{0}^{u_{M}} \frac{d \eta}{\sqrt{F\left(u_{M}\right)-F(\eta)}}$, where $u$ is a solution of (1.1), (1.2). We describe now a 
method which gives a lower bound under the assumption that

$$
f^{\prime}(u)<\lambda_{1} / \lambda,
$$

where $\lambda_{1}$ is given by (3.9). The specific result is as follows:

THEOREM IV. If $u$ is a positive classical solution of (1.1), (1.2) in a convex region $D$, then provided (6.1) is satisfied it follows that

$$
\left[\int_{0}^{u_{M}} \frac{d \eta}{\sqrt{F\left(u_{M}\right)-F(\eta)}}\right]^{2} \geqq \frac{\pi^{2} \lambda}{2 \lambda_{1}} .
$$

To establish this theorem we set

$$
\Phi=\cos \left[\sqrt{\frac{\lambda_{1}}{2 \lambda}} \int_{u}^{u_{M}} \frac{d \eta}{\sqrt{F\left(u_{M}\right)-F(\eta)}}\right]
$$

and form

$$
\begin{aligned}
\Delta \Phi+\lambda_{1} \Phi= & \frac{\sqrt{\lambda_{1}}}{2 \sqrt{2 \lambda\left[F\left(u_{M}\right)-F(u)\right]}}\left\{\sqrt{\frac{2 \lambda_{1}}{\lambda}} \sqrt{F\left(u_{M}\right)-F(u)} \cos \gamma_{1}-f(u) \sin \gamma_{1}\right\} \\
& \times\left\{2 \lambda-\frac{|\operatorname{grad} u|^{2}}{\left[F\left(u_{M}\right)-F(u)\right]}\right\}
\end{aligned}
$$

valid for $u \neq u_{M}$. Here we have used $\gamma_{1}$ to denote the argument of the cosine function in (6.3). The right hand side of (6.4) actually tends to zero as $u \rightarrow u_{\mathrm{M}}$. We show that in fact the right hand side of (6.4) is nonpositive if $f$ satisfies (6.1).

We first note that the last term in brackets is nonnegative by virtue of (2.1). To check the sign of the first term we denote this expression by $R$ and form

$$
\frac{d R}{d u}=\sin \gamma_{1}\left[\frac{\lambda_{1}}{\lambda}-f^{\prime}(u)\right]
$$

Thus either $\gamma_{1}>\pi$ or else

$$
\frac{d R}{d u} \geqq 0 .
$$

But the latter inequality implies that

$$
R(u) \leqq R\left(u_{M}\right)=0 .
$$

We thus conclude that either

$$
\int_{0}^{u_{M}} \frac{d \eta}{\sqrt{F\left(u_{M}\right)-F(\eta)}} \geqq \sqrt{2\left(\lambda / \lambda_{1}\right)} \pi
$$

or

$$
\Delta \Phi+\lambda_{1} \Phi \leqq 0 \text { in } D .
$$

By the arguments of Sections IV and V it follows that if $\Phi$ satisfies (6.9), then $\Phi$ cannot remain positive in $D$ and hence that $\Phi \leqq 0$ on $\partial D$. But this implies (6.2) which would also follow if $\gamma_{1}>\pi$. The theorem is thus established. Again the equality sign holds in the limit as $D$ degenerates to an infinite slab. 
If the inequality sign in (6.1) were reversed one could conclude that

$$
\Delta \Phi+\lambda_{1} \Phi \geqq 0 \text { in } D,
$$

but this inequality does not appear to be lead to very interesting results.

There are of course other more direct methods of comparing solutions of (1.1) to $\psi$ when $f(0) \neq 0$. In this case we may make the substitution

$$
w=\int_{0}^{u} \frac{d \eta}{f(\eta)}
$$

and observe that $w$ satisfies

$$
\begin{aligned}
\Delta w & =-\lambda-f^{\prime}(u)|\operatorname{grad} w|^{2} \text { in } D \\
w & =0 \text { on } \partial D .
\end{aligned}
$$

An immediate consequence of this expression (with $f$ satisfying (1.2)) is

$$
w \geqq \lambda \psi \text {. }
$$

Then if

$$
\int_{0}^{\infty} \frac{d \eta}{f(\eta)}=M<\infty
$$

it follows directly that no solution can exist if

$$
\lambda \psi_{M}>M
$$

This technique for obtaining criteria for nonexistence is well known.

Since (6.13) holds throughout $D$, and $w$ and $\psi$ both vanish on $\partial D$, it follows also that on $\partial D$

$$
|\operatorname{grad} w|=\frac{1}{f(0)}|\operatorname{grad} u| \geqq \lambda|\operatorname{grad} \psi| .
$$

Different results can sometimes be obtained, if we rewrite (6.12) replacing $|\operatorname{grad} w|^{2}$ on the right by $|\operatorname{grad} u|^{2} / f^{2}(u)$ and use (2.1), i.e.

$$
\begin{gathered}
\Delta w \geqq-\lambda\left[1+\frac{2 f^{\prime}(u)}{f^{2}(u)} F\left(u_{M}\right)-\frac{2 f^{\prime}(u)}{f^{2}(u)} F(u)\right] \text { in } D \\
w=0 \text { on } \partial D .
\end{gathered}
$$

If we are able to express the right hand side of (6.17) again in terms of $w$ then the inequality will sometimes yield upper bounds for $u$.

In the special case $f(u)=e^{u}$ we have

$$
w=1-e^{-u}
$$

and we conclude that

$$
1-e^{-u} \geqq \lambda \psi,
$$

from which it follows trivially that no solution exists if $\lambda \psi_{M}>1$. On the other 
hand if a solution exists then (6.17) becomes

$$
\begin{aligned}
\Delta w & \geqq-\lambda\left[\left(2 e^{u_{M}}-1\right)-2 e^{u_{M}} w\right] \text { in } D \\
w & =0 \text { on } \partial D .
\end{aligned}
$$

If $\tilde{\psi}$ satisfies (6.20) with the equality sign then clearly

$$
w \leqslant \tilde{\psi} \text { in } D .
$$

Again applying (2.1) to the function $\tilde{\psi}$ and integrating to the boundary we obtain

$$
\tilde{\psi}_{M} \leqq\left[1-\frac{e^{-u_{M}}}{2}\right]\left[1-\operatorname{sech} \sqrt{2 \lambda e^{u_{M}}} d\right]
$$

where $d$ is the radius of the largest inscribed $N$ sphere in $D$. Then the fact that $w_{M} \leqq \tilde{\psi}_{M}$ leads to a result implied already by $(3.13 \mathrm{~b})$, i.e.

$$
e^{u_{M} / 2} \leqq \cosh \sqrt{\frac{\lambda}{2}} e^{u_{M} / 2} d
$$

or setting $x=\sqrt{\frac{\lambda}{2}} e^{u_{\mathrm{M}} / 2} d$

$$
x \leqq \sqrt{\frac{\lambda}{2}} d \cosh x .
$$

Also since (6.21) holds throughout $D$ and both terms vanish on $\partial D$ we conclude that

$$
|\operatorname{grad} w|^{2}=|\operatorname{grad} u|^{2} \leqq|\operatorname{grad} \tilde{\psi}|^{2} \quad \text { on } \partial D .
$$

Applying (2.1) to the equation for $\tilde{\psi}$ we conclude that on $\partial D$

$$
|\operatorname{grad} \tilde{\psi}|^{2} \leqq 2 \lambda e^{u_{M}}\left[\left(2-e^{-u_{M}}\right)-\tilde{\psi}_{M}\right] \tilde{\psi}_{M} .
$$

Since $2 \tilde{\psi}_{M} \leqq 2-e^{-u_{M}}$ it follows from an application of (6.22) that

$$
|\operatorname{grad} \tilde{\psi}|^{2} \leqq \sqrt{\frac{\lambda}{2}}\left(2 e^{u_{M} / 2}-e^{-u_{M} / 2}\right) \tanh \left(\sqrt{2 \lambda} e^{u_{M} / 2} d\right), \quad \text { on } \partial D .
$$

Inserting this expression into (6.25) we conclude that

$$
\max _{\partial D}|\operatorname{grad} u| \leqq \sqrt{\frac{\lambda}{2}}\left(2 e^{u_{M} / 2}-e^{-u_{M^{\prime}} / 2}\right) \tanh \left(\sqrt{2 \lambda} e^{u_{M} / 2} d\right)
$$

whịch implies that

$$
\max _{\partial D}|\operatorname{grad} u| \leqq \sqrt{2 \lambda} e^{u_{M} / 2} .
$$

It seems likely that the results of this paper can be extended to handle more general classes of nonlinear second order elliptic equations, but up to now this has been done only for a few special cases. 


\section{References}

1 H. Amann. Fixed point equations and nonlinear eigenvalue problems in ordered Banach spaces. SIAM Rev. 18 (1976), 620-709.

2 C. Bandle. Existence theorems, qualitative results and a priori bounds for a class of nonlinear Dirichlet problems. Arch. Rational Mech. Anal. 58 (1975), 219-238.

3 S. Fu and L. Wheeler. Stress bounds for bars in torsion. J. Elasticity 3 (1973), 1-13.

$4 \mathrm{~J}$. Hersch. On the torsion function, Green's function and conformal radius: An isoperimetric inequality of Polya and Szegö, some extensions and application. J. Analyse Math. 36 (1980), $102-117$.

5 E. Hopf. A remark on elliptic differential equations of the second order. Proc. Amer. Math. Soc. 3 (1952), 791-793.

6 L. E. Payne. Bounds for the maximum stress in the Saint Vendnt torsion problem. Indian J. Mech. Math. Special Issue (1968), 51-59.

7 L. E. Payne and G. A. Philippin. Some maximum principles for nonlinear elliptic equations in divergence form with applications to capillary surfaces and to surfaces of constant mean curvature. Nonlinear Anal. 3 (1979), 193-211.

8 L. E. Payne and G. A. Philippin. On maximum principles for a class of nonlinear second order elliptic equations. J. Differential Equations 37 (1980), 39-48.

9 L. E. Payne and I. Stakgold. On the mean value of the fundamental mode in the fixed membrane problem. Applicable Anal. 3 (1973), 295-303.

10 M. H. Protter and H. F. Weinberger. Maximum principles in differential equations (Englewood Cliffs, N.J.: Prentice-Hall, 1967).

11 I. Stakgold and L. E. Payne. Nonlinear problems in nuclear reactor analysis. Proc. Conf. on Nonlinear Problems in the Physical Sciences and Biology. Lecture Notes in Mathematics 322, pp. 298-307 (Berlin: Springer, 1973).

12 H. F. Weinberger. Symmetrization in uniformly elliptic problems. Studies in Mathematical Analysis and Related Topics: Essays in honour of G. Polya, pp. 424-428 (Stanford, Calif.: Stanford Univ. Press. 1962).

(Issued 28 April 1981) 